\title{
Chainlike silicon nanowires: Morphology, electronic structure and luminescence studies
}

\author{
X. H. Sun \\ Department of Chemistry, University of Western Ontario, London N6A 5B7, Canada \\ N. B. Wong ${ }^{\text {a) }}$ \\ Center of Super-Diamond and Advanced Films (COSDAF), Department of Biology and Chemistry, City \\ University of Hong Kong, Hong Kong SAR, China \\ C. P. Li and S. T. Lee \\ Center of Super-Diamond and Advanced Films (COSDAF), Department of Physics and Materials Science, \\ City University of Hong Kong, Hong Kong SAR, China \\ T. K. Sham ${ }^{\text {b) }}$ \\ Department of Chemistry, University of Western Ontario, London N6A 5B7, Canada
}

(Received 22 December 2003; accepted 24 June 2004)

\begin{abstract}
The chainlike silicon nanowires SiNWs have been synthesized by fluctuating the pressure of the carrier gas in the growth process. The chainlike SiNWs comprise crystalline Si nanoparticles interconnected by amorphous silicon oxide wires. In addition to the sphere, other interesting shapes such as rectangular and triangular Si nanoparticles in chainlike SiNWs were also observed. X-ray absorption fine structure shows that the Si nanoparticles in the chainlike SiNWs are crystalline silicon and that the wire has a significantly larger $\mathrm{Si}$ oxide to crystalline $\mathrm{Si}$ ratio and disorder compared to the normal SiNW. X-ray excited optical luminescence and photoluminescence spectroscopy show that the relatively strong luminescence from the chainlike SiNWs compared to normal SiNWs arises mainly from silicon oxide in the chainlike SiNWs. (c) 2004 American Institute of Physics. [DOI: 10.1063/1.1782958]
\end{abstract}

\section{INTRODUCTION}

In recent years, considerable efforts have been made in the fabrication and characterization of silicon nanowires (SiNW)s because SiNW's exhibit different properties corresponding to bulk silicon and their potential application in future nanoelectronics. Many synthesis strategies to obtain bulk quantities of SiNWs have now been developed using both gas phase and condensed phase procedures. ${ }^{1-3}$ SiNWs show a variety of morphologies depending on the synthesis strategy and the growth conditions. ${ }^{4-8}$ It has been recognized that the electronic and optical properties of SiNWs exhibit morphology dependence, which may be employed for different fields of applications. ${ }^{9-15}$ One of the challenging issues in producing nanomaterials is to fabricate and control the morphology of the as-prepared nonostructures with a wellorganized arrangement, for example, equally spaced nanocrystallites and to specify their structural dependent electronic and optical properties. Unlike strong luminescence observed from porous silicon ${ }^{16}$ and other Si-based multilayer superlattices ${ }^{17}$ and nanoculsters, ${ }^{18}$ weak and complex luminescence from SiNWs with emission wavelength ranging from 420 to $820 \mathrm{~nm}$ have been observed due to the wide variation of morphology of SiNWs. ${ }^{12-15,19}$ The luminescence peaks have been ascribed variously to the quantum confinement effect, the defect centers surrounding the SiNW crys-

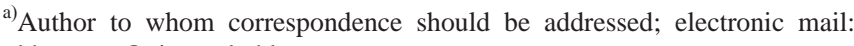
bhnwong@cityu.edu.hk

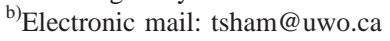

talline core, the silicon oxide surface, and the silicon-silicon oxide interface, or combinations thereof. In this paper, we report the synthesis of the chainlike silicon nanowires by simply fluctuating the pressure of the carrier gas during the growth of SiNWs, which exhibit unique and well-organized morphology comparing to the normal continuous SiNWs as revealed by high-resolution transmission electron microscopy (HRTEM). Further, we conducted a comparative study of the electronic and optical properties of the chainlike SiNW with those of the normal continuous SiNW using $\mathrm{x}$-ray absorption fine structure (XAFS), x-ray excited optical luminescence (XEOL), and photoluminescence (PL) spectroscopy. The XEOL technique ${ }^{20}$ monitors the optical response of a light-emitting material by tuning the $\mathrm{x}$-ray energy to a specific excitation channel (often at the x-ray absorption edge of a element of interest) and monitoring the luminescence with an optical monochromator (typically in a range of 200-900 nm). The relative quantum yield of the luminescence chromophore, which is either due to excitons, the recombination of a hole in the valence band and an electron in the conduction band or due to defects, depends strongly on the site and chemical environment of the absorbing atom. The photoluminescence yield (PLY) can in turn be used to record XAFS that provides structural information for the absorption site responsible for the luminescence..$^{20,21}$ Combining the XEOL and PLY spectra, we have been able to obtain the structural dependence of the luminescence. 


\section{EXPERIMENT}

The SiNWs used in this work were synthesized by thermal evaporation of $\mathrm{SiO}$ as previously described. ${ }^{4}$ The pressure in the system was kept oscillating in the range of 200-700 Torr in the growth process of SiNWs. The light yellow spongelike SiNWs were collected from the wall of the alumina tube where the temperature was approximately $930-950{ }^{\circ} \mathrm{C}$. The morphology and microstructure of the nanowire products were investigated by a Philips CM200 FEG HRTEM operating at $200 \mathrm{kV}$. The HRTEM samples were prepared by dispersing the product on a TEM grid with carbon holey film. The XAFS of the as-prepared chainlike SiNWs dispersed on a stainless steel substrate was carried out at the double crystal monochromator (DCM) beamline of the Canadian Synchrotron Radiation Facility (CSRF) located at the Synchrotron Radiation Center, University of Wisconsin-Madison. Total electron yield (TEY), fluorescence yield (FLY), and PLY were used to monitor the absorption. At these photon energies, TEY is sensitive to the surface and the near-surface region of the specimen while both FLY and PLY are sensitive to the bulk. The XEOL of the as-prepared chainlike SiNWs and their HF-etched samples excited with photon energy across the $\mathrm{Si} K$-edge were also obtained at the DCM beamline of CSRF, using a JY 100 monochromator to monitor the optical photons. A normal SiNW sample (smooth surface, $\sim 20 \mathrm{~nm}$ in diameter) was also studied for comparison. The PL of the as-prepared chainlike SiNWs and normal SiNWs were obtained by a micro-Raman spectrometer (Renishaw 2000 micro-Raman spectrometer) at room temperature. The $514.5 \mathrm{~nm}$ emission from an argon ion laser was used to excite the luminescence.

\section{RESULTS AND DISCUSSIONS}

The chainlike SiNWs had been previously observed as the by-product in the fabrication of normal SiNWs by both the laser ablation ${ }^{5}$ and thermal evaporation method. ${ }^{6}$ Bulk quantity of Si nanosphere chains had also been prepared by annealing normal SiNWs in high temperature via a spheroidization mechanism ${ }^{6,7}$ or by heating a gold-coated silicon substrate via an extension of the vapor-liquid-solid mechanism. ${ }^{8}$ These chainlike SiNWs were formed by silicon nanoparticles (actually, silicon nanospheres were observed in all previous studies), which are encapsulated and connected by a continuous outer layer of silicon dioxide. Figure 1 shows a typical TEM image of the chainlike SiNWs synthesized in this study. From Fig. 1, we see that the nanoparticles in the chainlike SiNWs have an average diameter of $16 \mathrm{~nm}$ and are connected by $\sim 20 \mathrm{~nm}$ long oxide wires. The morphology and microstructure of the chainlike SiNWs are shown in the HRTEM images in Fig. 2. Clearly, the nanoparticles are single silicon crystallites as confirmed by electron diffraction. The outer shells of the nanoparticles and interconnects are amorphous silicon oxide. It is interesting to note that the silicon nanoparticles in the chainlike SiNWs exhibit a variety of shapes. In addition to the sphere shape observed in previous studies, shown in Fig. 2(a), the rectangle and triangle shapes of silicon nanoparticles were also observed, as shown in Figs. 2(b) and 2(c), respectively. From the HR-

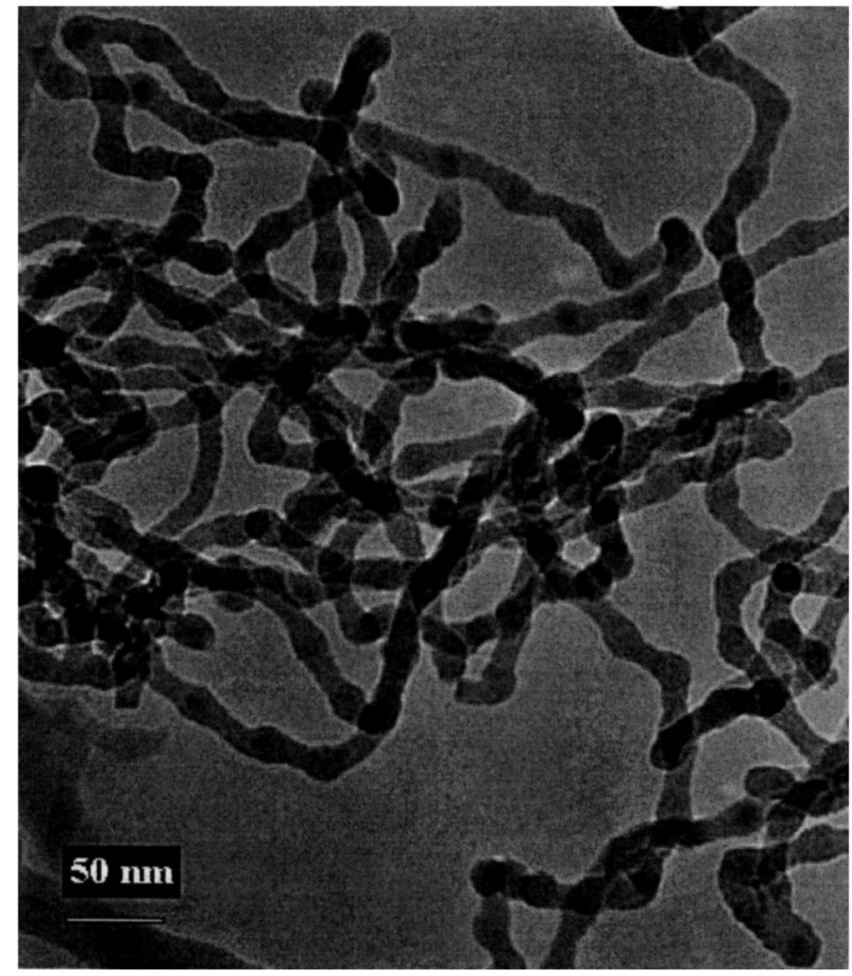

FIG. 1. A typical TEM image of the chainlike SiNW's.

TEM images, we find that the Si nanoparticles exhibit (111) crystal planes with a $3.1 \AA d$ spacing. In recently study, ${ }^{22}$ the horizontal cross section of a chainlike SiNW was obtained and it was found that some nanoparticles, measuring $16-20 \mathrm{~nm}$ in diameter, exhibited polyhedral shapes such as hexagons and octagons in the cross-sectional view. These observations are in contrast to previous studies in which only Si nanospheres were observed. Obviously, the mechanism of spheroidization of cylindrical nanowires of semi-infinite length at high temperature cannot give a satisfactory explanation for the formation of silicon nanoparticles with polyhedral shapes. We believe that the fluctuation of the pressure in the SiNW growth process is a main factor contributing to the formation of the chainlike SiNWs in our experiments. The details of the mechanism await further investigation.

Figure 3 shows the TEY and FLY Si $K$-edge XAFS of the as-prepared chainlike SiNW and normal SiNW specimens. The spectra have been normalized to unity edge jump and shifted vertically for clarity. All spectra exhibit a resonance at $1841 \mathrm{eV}$ and an intense resonance at $\sim 1847.5 \mathrm{eV}$. The first resonance at $\sim 1841 \mathrm{eV}$ arises form the crystalline $\mathrm{Si}$ nanoparticles in the chainlike SiNWs and the Si core of the normal nanowires. The intense resonance at $\sim 1847.4 \mathrm{eV}$ in all TEY, but a much-reduced intensity in all FLY, is characteristic of a $\mathrm{Si}$ is to $3 p$ ( $t_{2}$ orbital in a Td symmetry) transition of $\mathrm{SiO}_{2}$ and is due to the outer silicon oxide layer of the SiNWs. It is worthy noting that both chainlike SiNWs and normal SiNWs exhibit a larger intensity ratio of $\mathrm{Si}$ $(1841 \mathrm{eV})$ to $\mathrm{SiO}_{2}$ resonance $(1847.5 \mathrm{eV})$ in FLY than those in TEY. This is due to the fact that TEY is a surface sensitive technique, which is more sensitive to the surface oxide than the encapsulated $\mathrm{Si}$, whereas FLY is bulk sensitive. These results confirm that the oxide is on the surface of the nanow- 

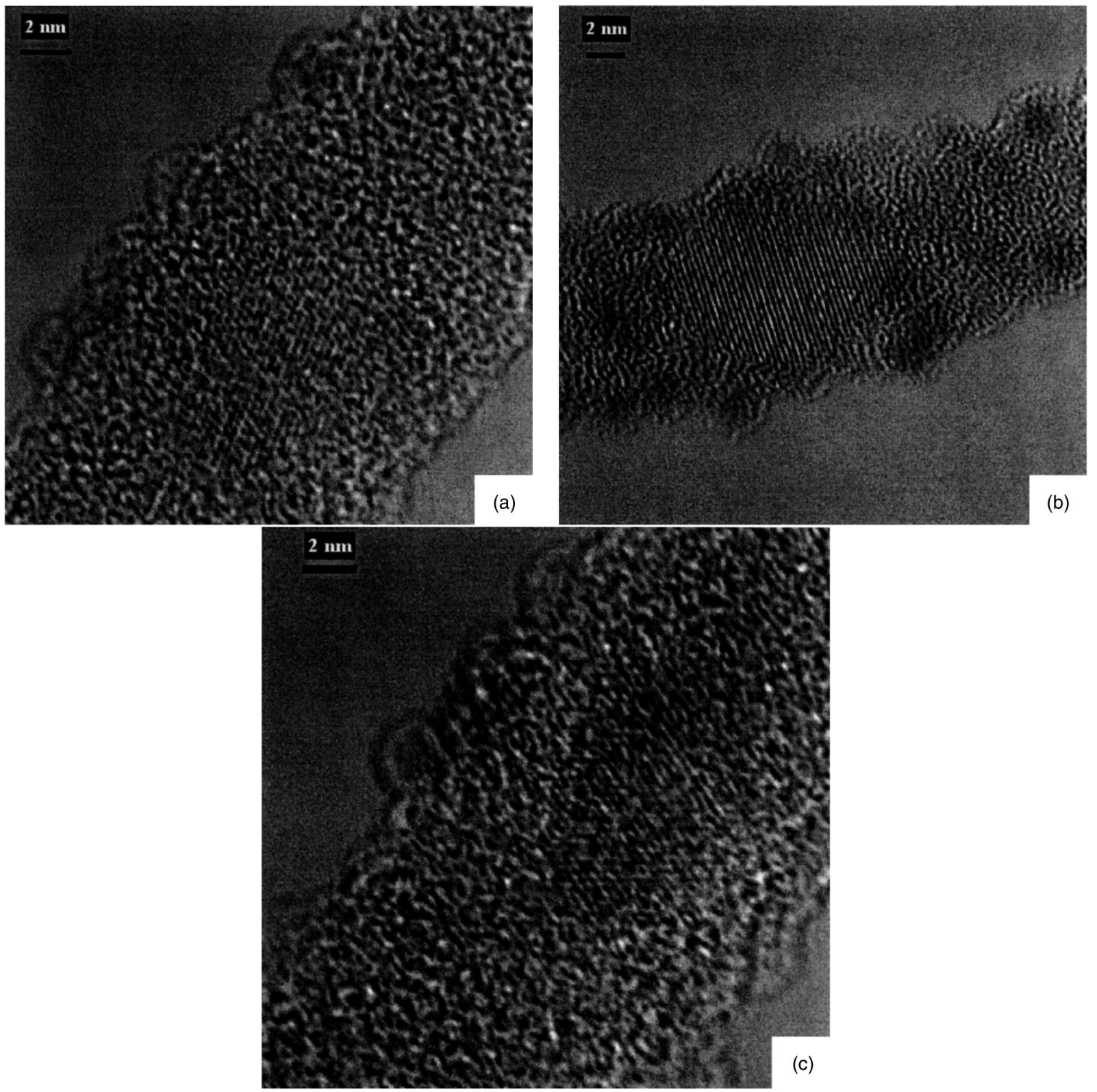

FIG. 2. The HRTEM images of the chainlike SiNW's. (a) A spherical Si nanoparticle, (b) a rectangular Si nanoparticle, and (c) a triangular Si nanoparticle, in the chainlike SiNW's, respectively.

ire. The perhaps most noticeable feature in Fig. 3 is that the chainlike SiNWs exhibit a much stronger $\mathrm{SiO}_{2}$ resonance, in both TEY and FLY than that of the normal SiNWs. This is due to a larger ratio of $\mathrm{Si}$ (oxide) atom to elemental atom in the former. This observation indicates that the nanoparticles in the chains are embedded entirely in silicon oxide as observed by HRTEM. Another feature worthy nothing in Fig. 3 is that the resonances between 1850 and $1900 \mathrm{eV}$ in $\mathrm{Si}$ $K$-edge result for the chainlike SINW shows silicon oxide dominant features.

The XEOL of the chainlike SiNWs compared to the normal SiNWs excited with photon energies at $\mathrm{Si} K$-edge $(1841 \mathrm{eV})$ and $\mathrm{SiO}_{2}$ resonance position $(1848 \mathrm{eV})$ is shown in. Fig. 4. Two emission bands in the green and blue regions peaking at $460 \mathrm{~nm}(2.7 \mathrm{eV})$ and $530 \mathrm{~nm}(2.34 \mathrm{eV})$, respectively are observed. The chainlike SiNWs exhibit much stronger emission intensity than the normal SiNWs. The in-

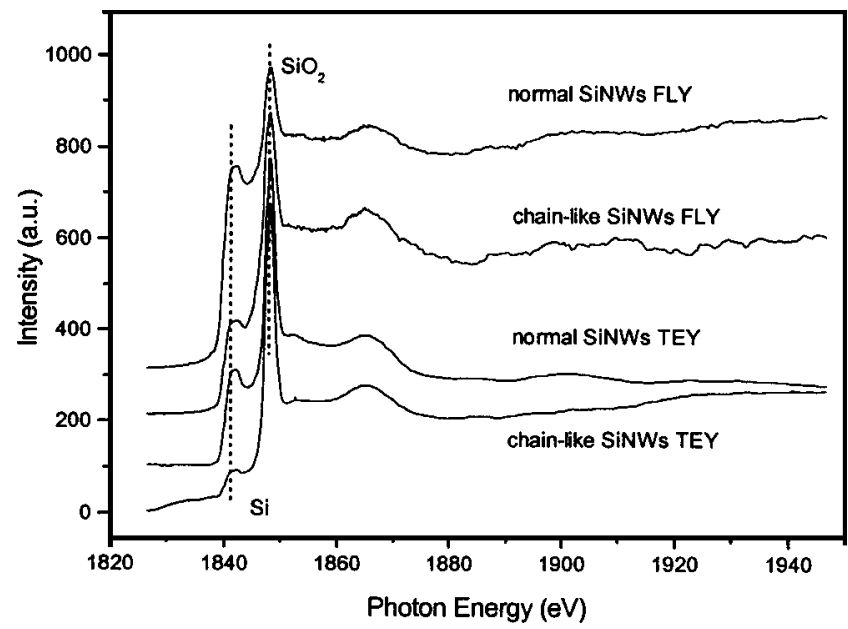

FIG. 3. TEY and FLY Si $K$-edge XAFS of the chainlike SiNW and normal SiNW specimens. 


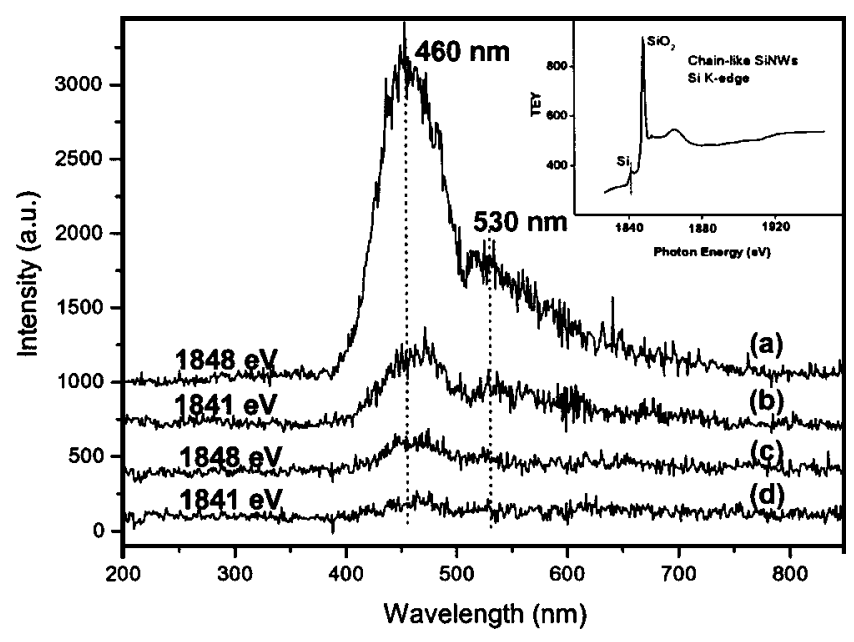

FIG. 4. XEOL of the chainlike SiNW's (curves a, b) compared to the normal SiNW's (curves c, d) excited with photon energies at $\mathrm{Si} K$-edge $(1841 \mathrm{eV})$ and $\mathrm{SiO}_{2}$ resonance position $(1848 \mathrm{eV})$.

tensities of the two peaks increased remarkably, especially in the chainlike SiNWs when the $K$-edge channel of silicon oxide turns on at $1847.5 \mathrm{eV}$. The peak at $2.7 \mathrm{eV}$ is similar to the results observed in the silica nanowires ${ }^{12}$ and can be attributed to the neutral oxygen vacancy in the oxide, while the peak at $2.34 \mathrm{eV}$ is less straightforward to assign. Close examination of the branching ratio (intensity of an individual emission over the overall emission intensity) of the two peaks in the chainlike SiNW spectra excited at 1841 and $1847.5 \mathrm{eV}$ reveals that the $530 \mathrm{~nm}$ emission is noticeably more intense at the $1841 \mathrm{eV}$ excitation (by 25\%). Also, when the silicon oxide channel turns on, the intensity increase of the peak at $2.34 \mathrm{eV}$ is less than that at $2.7 \mathrm{eV}$. It indicates that the $2.34 \mathrm{eV}$ peak is at least not completely associated with the $\mathrm{Si}$ oxide. It may arise primarily from the small $\mathrm{Si}$ crystallites embedded in the SiNW (according to the quantum confinement model, the crystallite size has to be smaller than $2 \mathrm{~nm}^{23-25}$ ) or from the defects surrounding the silicon nanoparticles. Thus the $530 \mathrm{~nm}$ emission is likely to have some quantum confinement origin. This is consistent with a recent observation. ${ }^{19}$

Figure 5 shows the PLY at the Si K-edge XAFS of the chainlike SiNW sample monitored with total (zero order) and wavelength-selected $(460 \mathrm{~nm})$ luminescence. The TEY and FLY are also shown. Both PLY spectra show similar spectral features to those observed in TEY and FLY spectra except for the intensity of the elemental $\mathrm{Si}$ whiteline $(\sim 1841 \mathrm{eV})$ which decreases noticeably in the zero-order PLY and nearly disappears at the $460 \mathrm{~nm}(2.7 \mathrm{eV})$ PLY. As discussed above, TEY is surface sensitive while FLY and PLY are bulk sensitive. This observation indicates that the total luminescence (zero-order) PLY arises mainly from silicon oxide as expected from Fig. 4. The disappearance of the elemental Si whiteline and an enhanced oxide feature in the $460 \mathrm{~nm}$ PLY show that the luminescence at $460 \mathrm{~nm}(2.7 \mathrm{eV})$ is primarily from silicon oxide. The weaker luminescence at $530 \mathrm{~nm}$ prevented the accumulation of satisfactory statistics for the $530 \mathrm{~nm}$ PLY measurement, but the difference in the intensity of the elemental $\mathrm{Si} K$-edge whiteline between the zero-order and $460 \mathrm{~nm}$ PLY clearly indicates that the $530 \mathrm{~nm}$

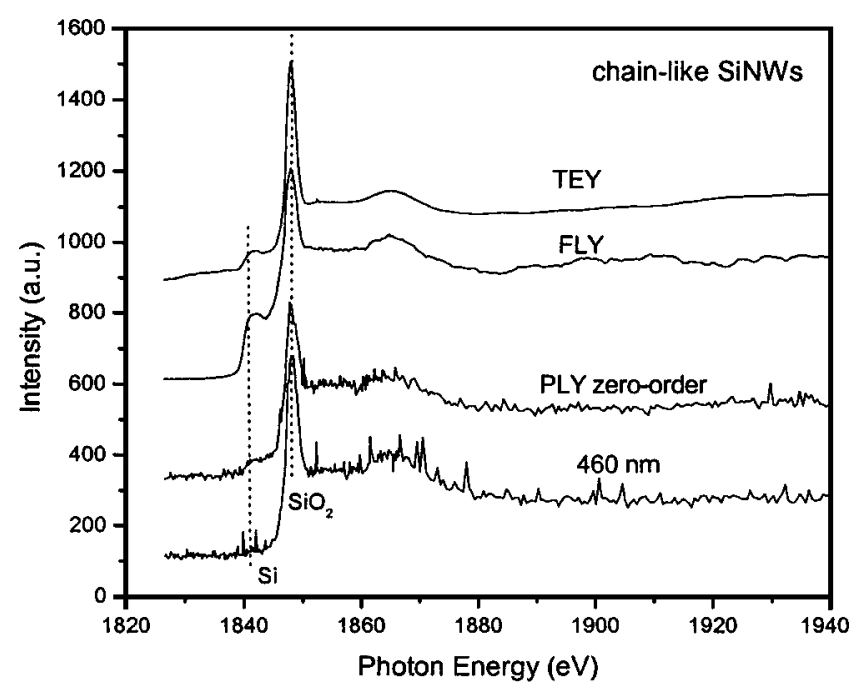

FIG. 5. PLY at the Si K-edge XAFS of the chainlike SiNW sample monitored with total (zero-order) and wavelength-selected $(460 \mathrm{~nm})$ luminescence. TEY and FLY are also shown.

one should at least have some contributions from the quantum-confined $\mathrm{Si}$ crystallites. Another evidence for the oxide dominant luminescence in chainlike SiNWs comes from XEOL recorded after the complete removal of silicon oxide by HF solution. After HF-etching, no luminescence can be observed in either chainlike SiNWs or normal SiNWs under the same experimental conditions.

Finally, the PL spectra of the chainlike and normal SiNWs excited at $514.5 \mathrm{~nm}$ with an argon ion laser are shown in Fig. 6. The PI spectral features for both samples are similar except for the much stronger emission in the chainlike SiNW. A strong PL peak around $560 \mathrm{~nm}$ with a broad shoulder at $620 \mathrm{~nm}$ was obtained for the chainlike SiNW sample. We attribute the PL to the defects in silicon oxide or interface between silicon oxide and nanoparticles. It is interesting to note that the $460 \mathrm{~nm}$ emission observed in XEOL is not accessible with the laser.

In conclusion, the chainlike silicon nanowires synthesized by fluctuating the pressure in the process of growth of

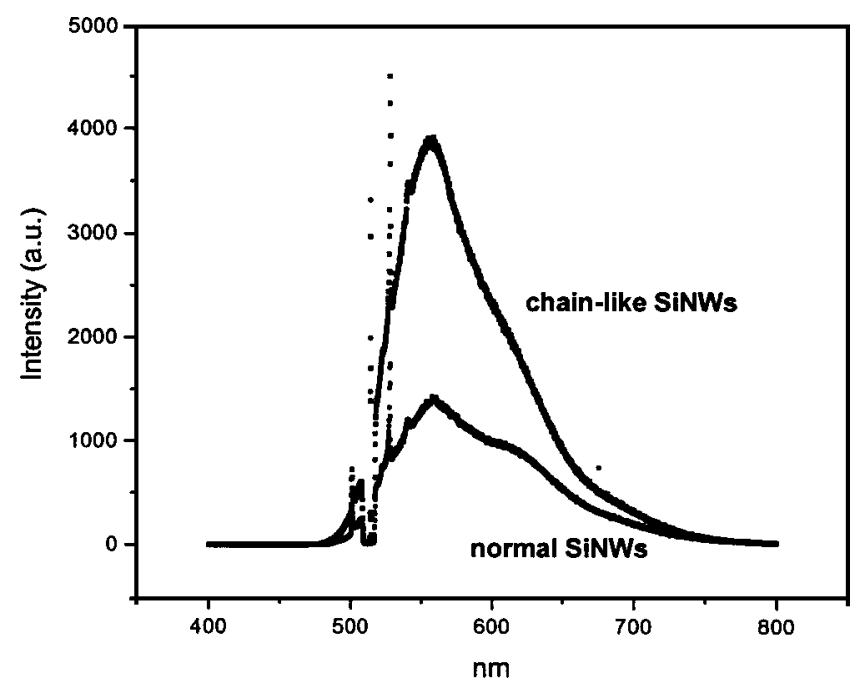

FIG. 6. PI spectra of the chainlike and normal SiNW's excited at $514 \mathrm{~nm}$ with an argon ion laser. 
SiNWs are reported. HRTEM shows that the chainlike SiNWs comprise crystalline Si nanoparticles of an average diameter of $16 \mathrm{~nm}$ and interconnecting $20 \mathrm{~nm}$ long silicon oxide wires. In addition to at the sphere, rectangle and triangle shapes of Si nanoparticles in the chainlike SiNWs were observed. XAFS studies reveal a significantly larger ratio of $\mathrm{Si}$ in oxide to $\mathrm{Si}$ in elemental crystallite in the chainlike SiNWs consistent with the HRTEM observation. XEOL and PL results show that the major portion of the luminescence from the chainlike SiNW arises primarily from the silicon oxide while only a minor component is from quantumconfined silicon luminescence, in contrast to porous silicon luminescence.

\section{ACKNOWLEDGMENTS}

Research at the University of Western Ontario was supported by the Natural Science and Engineering Research Council (NSERC) of Canada. CSRF was supported by NSERC through a MFA grant and the National Research Council (NRC) of Canada. SRC was supported by the U.S. National Science Foundation under Grant No. DMR-0084402. N. B. W. acknowledges the support of a grant from the Research Grants Council of Hong Kong SAR [SiNWs RGC Grant 9040879 (CityU 1024/03)].

${ }^{1}$ Y. F. Zhang, Y. H. Tang, N. Wang, D. P. Yu, C. S. Lee, I. Bello, and S. T. Lee, Appl. Phys. Lett. 72, 1835 (1998).

${ }^{2}$ A. M. Morales and C. M. Lieber, Science 279, 208 (1998).

${ }^{3}$ D. P. Yu, Z. G. Bai, Y. Ding, Q. L. Hang, H. Z. Zhang, J. J. Wang, Y. H. Zou, W. Qian, G. C. Xiong, H. T. Zhou, and S. Q. Feng, Appl. Phys. Lett. 283, 3458 (1998).

${ }^{4}$ W. S. Shi, H. Y. Peng, Y. F. Zheng, N. Wang, N. G. Shang, Z. W. Pan, C.
S. Lee, and S. T. Lee, Adv. Mater. (Weinheim, Ger.) 12, 1343 (2000).

${ }^{5}$ Y. H. Tang, Y. F. Zhang, N. Wang, C. S. Lee, X. D. Han, I. Bello, and S. T. Lee, J. Appl. Phys. 85, 7981 (1999)

${ }^{6}$ H. Y. Peng, Z. W. Pan, L. Xu, X. H. Fan, N. Wang, C. S. Lee, and S. T. Lee, Adv. Mater. (Weinheim, Ger.) 13, 317 (2001).

${ }^{7}$ H. Y. Peng, N. Wang, W. S. Shi, Y. F. Zhang, C. S. Lee, and S. T. Lee, J. Appl. Phys. 89, 727 (2001).

${ }^{8}$ H. Kohno and S. Takeda, Appl. Phys. Lett. 73, 3144 (1998).

${ }^{9}$ S. T. Lee, N. Wang, Y. F. Zhang, and Y. H. Tang, MRS Bull. 24, 36 (1999).

${ }^{10}$ Y. Cui, X. F. Duan, J. T. Hu, and C. M. Lieber, J. Phys. Chem. B 104, 5213 (2000).

${ }^{11}$ G. W. Zhou, H. Li, D. P. Yu, Y. Q. Wang, X. J. Huang, L. Q. Chen, and Z. Zhang, Appl. Phys. Lett. 75, 2447 (1999).

${ }^{12}$ D. P. Yu, Q. L. Hang, Y. Ding, H. Z. Zhang, Z. G. Bai, J. J. Wang, Y. H. Zou, W. Qian, W. Qian, G. C. Xiong, and S. Q. Feng, Appl. Phys. Lett. 73, 3076 (1998).

${ }^{13}$ J. Qi, A. M. Belcher, and J. M. White, Appl. Phys. Lett. 82, 2616 (2003).

${ }^{14}$ C. Wu, W. Qin, G. Qin, D. Zhao, J. Zhang, W. Xu, and H. Lin, Chem. Phys. Lett. 378, 368 (2003).

${ }^{15}$ S. T. Lee, N. Wang, and C. S. Lee, Mater. Sci. Eng., A 286, 16 (2000).

${ }^{16}$ L. T. Canham, Appl. Phys. Lett. 57, 1046 (1990).

${ }^{17}$ Z. H. Lu, D. J. Lookwood, and J. M. Barbeau, Nature (London) 378, 258 (1995).

${ }^{18}$ J. P. Wilcox and G. A. Samara, Appl. Phys. Lett. 74, 3164 (1999).

${ }^{19}$ T. K. Sham, S. J. Naftel, P.-S. G. Kim, R. Sammyraiken, Y. H. Tang, I. Coulthard, A. Moewes, J. W. Freeland, Y. F. Hu, and S. T. Lee, Phys. Rev. B 70, 045313 (2004).

${ }^{20}$ T. K. Sham, D. T. Jiang, I. Coulthard, J. W. Lorimer, X. H. Feng, K. H. Tan, S. P. Frigo, R. A. Rosenberg, D. C. Houghton, and B. Bryskiewicz, Nature (London) 363, 331 (1993).

${ }^{21}$ S. J. Naftel, P.-S. G. Kim, T. K. Sham, R. Sammynaiken, B. W. Yates, and Y.-F. Hu, J. Appl. Phys. 93, 5191 (2003).

${ }^{22}$ B. K. Teo, X. H. Sun, T. F. Hung, X. M. Meng, N. B. Wong, and S. T. Lee, Nano Lett. 3, 1735 (2003).

${ }^{23}$ S. Ogut, J. R. Chelikowsky, and S. G. Louie, Phys. Rev. Lett. 79, 1770 (1997).

${ }^{24}$ G. Allan, C. Delerue, and M. Lannoo, Phys. Rev. Lett. 76, 2961 (1996).

${ }^{25}$ P. E. Batson and J. R. Heath, Phys. Rev. Lett. 71, 911 (1993). 